Published in final edited form as:

Behav Res Ther. 2013 June ; 51(6): 256-265. doi:10.1016/j.brat.2013.01.010.

\title{
Subjective Experience of Sensation in Anorexia Nervosa
}

\author{
Nancy L. Zucker ${ }^{a, b}$, Rhonda M. Merwin ${ }^{a}$, Cynthia M. Bulik ${ }^{c}$, Ashley Moskovich ${ }^{b}$, Jennifer \\ Wildes $^{d}$, and Jennifer Groh $^{b}$ \\ aDuke University Medical Center \\ bDuke University \\ cUniversity of North Carolina at Chapel Hill \\ dUniversity of Pittsburgh Medical Center
}

\begin{abstract}
The nature of disturbance in body experience in anorexia nervosa (AN) remains poorly operationalized despite its prognostic significance. We examined the relationship of subjective reports of sensitivity to and behavioral avoidance of sensory experience (e.g., to touch, motion) to body image disturbance and temperament in adult women currently diagnosed with AN $(n=20)$, women with a prior history of AN who were weight restored $(n=15)$, and healthy controls with no eating disorder history $(n=24)$. Levels of sensitivity to sensation and attempts to avoid sensory experience were significantly higher in both clinical groups relative to healthy controls. Sensory sensitivity was associated with body image disturbance $(r(56)=.51, p<.0001)$, indicating that body image disturbance increased with increased global sensitivity to sensation. Sensory sensitivity was also negatively and significantly correlated with lowest BMI $\left(r^{2}=-.32, p<.001\right)$, but not current BMI $\left(r^{2}=.03, p=.18\right)$, and to the temperament feature of harm avoidance in both clinical groups. We discuss how intervention strategies that address sensitization and habituation to somatic experience via conditioning exercises may provide a new manner in which to address body image disturbance in AN.
\end{abstract}

\section{Keywords}

anorexia nervosa; eating disorders; body image disturbance; kinesthesia; vestibular

Disturbance in the experience of the body is a defining feature of anorexia nervosa (AN). Historically, body image disturbance has been parsed along dimensions of cognition, perception, and experience (Gleaves, Williamson, Eberenz, Sebastian, \& Barker, 1995; Mohr et al., 2010)._ENREF_3 However, as elegantly summarized by Park, Dunn, and Barnard (2011), the vast majority of research on body image disturbance in AN has examined cognitive components, such as body dissatisfaction and perceptual aspects such

\footnotetext{
(C) 2013 Elsevier Ltd. All rights reserved.
}

Contact Information: Nancy Zucker, P.O. Box 3842, Department of Psychiatry and Behavioral Science, Duke University Medical Center, Durham, NC 27710, Phone: 919-668-2281, Fax: 919-681-7347, zuckeoo1@ mc.duke.edu.

Author Note: Drs. Zucker, Ms. Moskovich, and Dr. Groh are part of the Department of Psychology and Neuroscience at Duke

University. Drs. Zucker and Merwin are part of the Department of Psychiatry and Behavioral Sciences of Duke University Medical Center. Dr. Bulik is part of the Department of Psychiatry, University of North Carolina at Chapel Hill. Dr. Wildes is part of the Department of Psychiatry, University of Pittsburgh School of Medicine.

Publisher's Disclaimer: This is a PDF file of an unedited manuscript that has been accepted for publication. As a service to our customers we are providing this early version of the manuscript. The manuscript will undergo copyediting, typesetting, and review of the resulting proof before it is published in its final citable form. Please note that during the production process errors may be discovered which could affect the content, and all legal disclaimers that apply to the journal pertain. 
visual image distortion, but has neglected the subjective experience of the body. Subjective body experience is a broad domain that includes interoceptive, exteroceptive, vestibular, and proprioceptive inputs and thus extends from sensations within the body (e.g., a heartbeat) to sensations that define and impinge upon body boundaries (e.g., limb position, pressure on the skin). Characterizing disturbances in subjective body experience among individuals with AN is critical to understanding and altering the pathophysiology of this illness, as both the relentless pursuit of an unhealthy body weight and the disordered eating behaviors that typify AN may be motivated, in part, by a desire to alter body experience - not merely body appearance (Cserjesi et al., 2010; Sachdev, Mondraty, Wen, \& Gulliford, 2008).

Furthermore, body image disturbance has prognostic significance, predicting illness onset, maintenance, and remission (Fairburn et al., 2003; Jacobi, Hayward, de Zwaan, Kraemer, \& Agras, 2004; Keel, Dorer, Franko, Jackson, \& Herzog, 2005; Killen et al., 1994). Thus, study of the experiential aspects of body image disturbance in AN has the potential to guide the development of novel intervention approaches to address this pernicious symptom that eludes change.

Sensation constitutes the foundation of one's subjective body schema (Fisher, Cleveland, \& Davis, 1957; Gallagher \& Cole, 1995; Watanabe, 2005). As such, aberrations in basic sensory processes such as proprioception and kinesthesia (processes responsible for sensing the position of the body in space and the boundaries that define the body's limits respectively) may contribute to the disturbance of body experience in AN (Guimon, 1997). Similarly, capacities to sense pressure on the skin and the ability to integrate sensations, as in what one feels and what one sees, all play a role in constituting a cohesive sense of one's body boundaries (Anema et al., 2009; Gallagher \& Cole, 1995; Kammers, de Vignemont, Verhagen, \& Dijkerman, 2009). These basic processes may be or become disrupted in those with AN. Indeed, the role of sensation in contributing to or constituting body image disturbance has long informed research in AN, and was first postulated by Bruch in the realm of interoceptive signals (Bruch, 1962). More recently, atypical sensory experiences are increasingly suggested by the results of neuroimaging paradigms focused on selfreflection (Mohr, et al., 2010), self-perception (Sachdev, et al., 2008; Seeger, Braus, Ruf, Goldberger, \& Schmidt, 2002; Vocks et al., 2010), and perception of bodies of others (Sachdev, et al., 2008). Studies of basic proprioceptive or kinesthetic processes, however, are lacking with some noteworthy exceptions (e.g., Keizer et al., 2011).

Sensation is dynamic (Thompson, 2009). Thus, understanding the contribution of sensation to body image disturbance requires information about of how individuals with AN notice and adapt to changing bodily experience. Two processes that characterize such change are habituation and sensitization. In brief, habituation refers to decreased awareness of or responsiveness to a persistent or repeating stimulus (Thompson, 2009). The process of habituation is efficient in that it allows an individual to ignore sensory stimuli after they are initially registered (Thompson, 2009). The clinical presentation of AN suggests slower habituation to visceral experience, at least in certain illness relevant contexts, such as difficulty habituating to the sensation of fullness from the gut (Halmi \& Sunday, 1991; Radomsky, de Silva, Todd, Treasure, \& Murphy, 2002)._ENREF_22 There also is evidence that perpetual awareness of body schema impinges on adaptive functioning in individuals with AN. For example, the impaired concentration noted in AN may be related to altered somatic sensations, not just cognitive concerns related to weight and shape. Characterization of such subjective sensory experiences may provide novel interpretations for the motivations underlying frequently associated behavioral patterns in AN. For example, the tendency to wear loose clothing in AN may be less about concealing low body weight and more about aversion to the experience of tactile sensations (e.g., the feeling of clothing touching the skin or being tight or restricting). Similarly, perpetual awareness of the body may be less about overvaluation of thinness and more about aberrations in habituation to a proprioceptive or 
kinesthetic sense (e.g., visual and tactile body checking may reflect deficits in habituation rather than overvaluation). This is in contrast or in addition to cognitive accounts that ascribe such sensation to attitudinal factors which are informed by the current culture and may better be understood as epiphenomena of AN (Shafran \& Robinson, 2004).

In contrast to habituation, sensitization is a process whereby individuals' exhibit enhanced responsiveness to specific stimuli relative to their baseline experience with those stimuli. Thus, habitation and sensitization work in concert: individuals may be primed to differentially notice certain salient stimuli and then habituate to such stimuli if not relevant to ongoing motivations. For example, using a series of thermal stimuli that variously increased or decreased in intensity, Vierck et al.(2010) found that individuals were differentially sensitized to experience a sensation as more or less intense based on their immediate prior sensory experiences. In the context of chronic pain, robust evidence of lowered pain thresholds suggest that individuals who experience a chronic painful stimulus are more sensitive to the presence of novel sensory stimuli (Martucci, Yelle, \& Coghill, 2012; Nickel, Seifert, Lanz, \& Maihofner, 2012). The state of starvation, and associated low body weight, has been robustly reported to alter sensory experience in animal models and similarly may influence the experience of the body in AN (Wang, Hung, \& Randall, 2006). Critically, the ill state of AN is often sought by those affected. Thus, understanding the relationship of body mass index (BMI) to sensory experiences of sensitization and habituation may highlight why the ill state is reinforcing for those with AN. For example, a low BMI may mute aversive sensory experiences. In general, decreased sensitization would be maladaptive in that an individual would be less able to respond to the demands of a given moment. However, sensory changes may prove subjectively adaptive if such changes make the individual "feel" better. Relevant clinical examples include decreased sensitization to hunger and increased sensitivity to smell - adaptations that may facilitate survival of a starving animal but also may perpetuate the ill state of AN (Colbert \& Bargmann, 1997; Halmi \& Sunday, 1991; Korbonits, Blaine, Elia, \& Powell-Tuck, 2007; LeBoeuf, Guo, \& Garcia, 2011).

To be sure, the processes of sensitization and habituation are also subject to complex subjective interpretation, i.e., putative top-down influences that impact the interpretation of sensory experience. Motivation affects sensation far earlier in the temporal chain of sensory experience than previously documented (Laycock, Crewther, \& Crewther, 2007). For examples, visually guided attention is captured by what is motivationally salient and prior learning history affects the meaning ascribed to visceral and exteroceptive inputs (Fecteau \& Munoz, 2006). An individual may ascribe a temporary bloated feeling in the gut to excess salt from popcorn, their premenstrual status, or weight gain. Alternatively, individual differences in awareness of changes in the body may influence attention allocation. Thus, study of the role of sensation in contributing to or constituting body image disturbance in AN is complicated by the influence of motivation on perception. Combined this argues that both objective sensory thresholds and subjective interpretations are needed to fully understand the nature of somatic experience in AN.

Finally, sensation motivates behavior (Thompson, 2009). Individuals actively seek to alter their sensory experience, a motivation that may relate to models of homeostasis or allostasis in achieving a relatively stable sense of arousal (McEwen, 2004). Thus, in documenting a person's sensory experience, it is important not only to characterize his/her sensitivity to and interpretation of sensation, but also whether s/he actively seeks or avoids sensation. For example, an individual who is very sensitive to specific visual stimuli (e.g., scary movies), but actively seeks such experiences may be very different from an individual with matching sensory capacities who actively avoids such sensation. Such complex, temporal influences on sensory experience suggest that subjective body experience may be integrally related to 
temperament. In fact, organizational schemes of childhood temperament have been forged on a child's capacity and tendency to sense, regulate, seek, or avoid sensory experience (Beauchaine, Gatzke-Kopp, \& Mead, 2007; Gray, 1970; Moehler et al., 2006; RimmKaufman \& Kagan, 2005). In the field of eating disorders, the biologically-based temperament classification scheme of Cloninger has been the most researched (Cloninger, 1986). Within this framework, harm avoidance has been repeatedly documented in those with AN (Klump et al., 2000; Klump et al., 2004). Harm avoidance has largely been operationalized in the behavioral domain (i.e., actively avoiding uncertain circumstances). However, the research foundation laid by temperament research in children suggests that those with elevated harm avoidance may also experience enhanced sensory sensitivity and that behavioral avoidance may actually be secondary to or reinforcing of sensory sensitivity (Ben-Sasson, Carter, \& Briggs-Gowan, 2009; Goldsmith, Van Hulle, Arneson, Schreiber, \& Gernsbacher, 2006). If so, consistent findings of harm avoidance in those with AN may be related to more basic processes of perception.

In summary, subjective awareness of and behavioral responses to sensory experiences may be important contributors to the sensation and experiential aspects of body image disturbance in AN. Thus, in this study, we used systematic subjective reports of the experience of sensitivity to sensory input to better understand sensory experience in adults who were currently ill with AN (AN) and those with AN by history who were currently weight-restored (AN-WR), relative to typically developing controls. The inclusion of weight-restored individuals allows for the direct examination of the influence of weight on sensory sensitivity in comparison to the currently and never ill groups. We hypothesized that 1) both AN and AN-WR would endorse greater sensitivity to sensation (as a global measure) and report more frequent attempts to avoid sensory experiences relative to healthy controls, 2) such sensitivity to sensation would be positively associated with both body image disturbance and BMI, and 3) this sensitivity to sensation would also be positively correlated with the trait feature of harm avoidance. Finally, in a series of exploratory analyses, we examine differences in sensitivity to specific sensations (visual, touch, movement) across groups.

\section{Method}

\section{Participants}

Individuals were recruited via printed advertisements placed within a 50-mile radius of the hosting university, and via electronic advertisements posted to parent forums and web-sites devoted to eating disorders. Notices were sent to a mailing list of healthcare providers known to specialize in the care of individuals with eating disorders within a 60-minute traveling distance, to all therapists in two university-based eating disorder programs, and were posted in clinic waiting rooms. From a recruitment sample of 164 individuals, 99 passed the initial study screening criteria, and 74 met predetermined eligibility criteria (see below) and fifty-nine agreed to participate. Fifty-nine adult female participants were classified as follows: current diagnosis of AN (AN: $n=20$ ), weight-restored with a prior diagnosis of AN (AN-WR: $n=15$ ), and healthy controls with no history of AN (CN: $n=$ 24).

Eligibility Criteria-To be eligible, clinical individuals had to have met diagnostic criteria for AN (excluding the amenorrhea criterion consistent with prior studies (Roberto, Steinglass, Mayer, Attia, \& Walsh, 2008) based on the Diagnostic and Statistical Manual for Mental Disorders IV (American Psychiatric Association, 1994) either currently or in the past. In addition, it was also required that individuals were not actively experiencing psychosis or related thought disorder and were not actively abusing substances. Further, the 
current protocol was part of a larger study examining social cognition and interpersonal functioning in AN during which a comprehensive cognitive battery was administered. Given the potential influence of learning disabilities on cognitive outcomes, individuals with a diagnosed learning disability were excluded. Control participants had no history of eating disorder, in addition to meeting the eligibility criteria listed above. Participants completed an initial telephone interview to assess eating disorder history, followed by a face-to-face structured diagnostic interview of eating disorder symptoms over the past 3 months. This interview was conducted by a PhD-level clinical psychologist with extensive experience in assessing eating disorders.

BMI Determination-Maintenance of an unhealthy low-weight is a defining feature of AN. Although the DSM-IV provides an example of a low-weight threshold (e.g., less than $85 \%$ of ideal body weight), the determination of ideal body weight for a given individual is complex and is most precisely determined based on an individual's weight history. An individual's ideal body weight is the weight range associated with optimal health. Definition of a "less than ideal" body weight would then be defined as less than the lowest point of this healthy weight range. However, body mass index (BMI) itself is inadequate for indexing physical recovery given recent studies demonstrating that other physiological parameters such as insulin or degree of body fat, not body mass, were predictive of return of menstruation (Dei, Seravalli, Bruni, Balzi, \& Pasqua, 2008; Misra et al., 2006).

To determine weight thresholds for the current study, we combined information from a variety of sources: medical record abstraction (when further clarification was needed), a detailed weight history, structured diagnostic interview, and self-report measures. To do so, we follow a general algorithm to define ideal body weight in adults. First, via structured interview, we attempt to establish the weight the participant has most consistently maintained without attempts to restrict calories, engage in excessive or unhealthy weight loss behaviors, and during which they had a regular menstrual cycle and no other signs of medical compromise. Second, if the individual is not a good historian or if such a period did not exist, we then go to their medical record to discern the weight and height percentiles they most consistently maintained prior to eating disordered pathology. Third, once we find this percentile, we define ideal weight as a 5 pound range around this value. Fourth, unhealthy low weight is then defined based on percentages relative to the lower bound of this range, using $85 i l e \%$ as a starting point, but taking into account the bounds at which the individual demonstrates medical compromise. We defined weight-restoration as being at an individual's ideal body weight for $\geq$ six months. Thus, this strategy may lead to deviations from the classic distinction of a body mass index of 18.5 or less as underweight, but more accurately reflects the weight history and optimal functioning of a given individual. This approach is less arbitrary and more accurately reflects the inter-individual clinical complexity of AN.

\section{Procedure}

Participants completed a battery of neuropsychological measures and self-report measures of subjective sensory experience as part of a study of social cognition and interpersonal functioning in AN. Tasks were administered in a fixed order with neuropsychological measures interspersed with measures of sensory experience and diagnostic interviews to maintain interest and decrease participant burden. Self-report measures were chosen to characterize subjective sensory experience; body image disturbance; disordered eating behaviors and attitudes; symptoms of psychiatric disorders often comorbid in those with eating disorders; and more long-standing features of temperament. Both clinicianadministered structured interview and a self-guided structured interview of eating disorder history were used to characterize illness trajectories. The latter was based on a compilation 
of existing, well-validated, and frequently used semi-structured diagnostic interviews of eating disorder pathology, for example, the Structured Interview of Anorexia and Bulimia (SIAB; Fichter et al., 1991) and Eating Disorder Examination (EDE; Cooper \& Fairburn, 1987). From the SIAB, graphic illustrations were used to facilitate descriptions of the illness course of individual symptoms. Additional features included subjective assessment of time since the initiation of a symptom, subjective assessment of accuracy of illness history, and the inclusion of a validated measure of quality of life related to eating disorder history. Testing sessions were kept to a maximum of 3 hours and participants were offered breaks between every task. All protocols were approved by the Institutional Review Board of Duke University Medical Center (Pro00008689) and that of the University of North Carolina, Chapel Hill (Pro 07-1743).

\section{Assessment Measures}

Sensory Profile, Adolescent and Adult Report Version-(SP;Dunn, 1999). The SP is a widely used and validated measure of the subjective experience of sensation across multiple sensory domains as well as the behavioral response to sensation. The development of the SP is grounded in a neurological framework and subscales are designed to assess dimensions of habituation and sensitization by asking individuals how rapidly they notice and accommodate to sensations across sensory domains. Individuals characterize their perceived sensitization and habituation (Sensory Sensitivity; Low Registration) to experiences in the visual, auditory, touch, taste, smell, vestibular, and kinesthetic domains. Reactions to sensation in each domain are characterized by delineating those individuals who seek sensation (Sensation Seeking) from those who avoid sensation (Sensation Avoiding). The SP generates omnibus scores collapsed across sensations for Sensory Sensitivity, Low Registration, Sensation Seeking, and Sensation Avoidance. It is also possible to characterize experience and behavior related to a particular sensation (Kern et al., 2007). We employ both approaches. Evidence of construct validity,(Pfeiffer, Kinnealey, Reed, \& Herzberg, 2005) reliability, and discriminant validity have been established (Chen, Rodgers, \& McConachie, 2009; Gabriels et al., 2008).

Eating Disorder Examination Questionnaire-(EDE-Q; Fairburn \& Beglin, 1994) The EDE-Q is a 41-item self-report version of the EDE (Fairburn \& Cooper, 1993). Similar to the EDE, the EDE-Q measures eating disorder psychopathology and yields the same diagnostic criteria and four subscales scores. Normative data collected from a large sample of young adult women $(n=5231)$ between 18 and 42 years of age produced the following subscale means: Restraint $(M=1.30, S D=1.40)$, Eating Concern $(M=0.76, S D=1.06)$, Weight Concern $(M=1.79, S D=1.51)$, and Shape Concern $(M=2.23, S D=1.65)$. Good convergence between the EDE and EDE-Q has been documented among community and clinical samples, though inconsistencies have been reported (Binford, Le Grange, \& Jellar, 2005; Fairburn \& Beglin, 1994; Mond, Hay, Rodgers, Owen, \& Beumont, 2004)._ENREF_ 47 The internal consistency of the EDE-Q has been supported in clinical and college undergraduate populations (e.g., Luce \& Crowther, 1999; Peterson et al., 2007)_ENREF_60 and acceptable concurrent validity, criterion validity, and test-retest reliability have been documented (Luce \& Crowther, 1999; Mond, et al., 2004). We administered both measures to ensure that we captured the breadth of eating pathology; in particular, we feared that individuals might not disclose certain forms of pathology during a live interview.

Eating Disorder Examination-(EDE; Fairburn \& Cooper, 1993) The EDE is a widely implemented and favored structured diagnostic interview of eating disorder psychopathology (Garner, 2002). Behavioral and attitudinal features are assessed according to DSM-IV diagnostic criteria. While the interview largely focuses on symptoms over the past 28 days, behavioral diagnostic criteria (e.g., binge-eating, self-induced vomiting, driven 
exercise, laxative abuse) are assessed for presence, frequency, and total days of occurrence over the past three months in accordance with the duration criteria specified in DSM-IV eating disorder diagnoses. Attitudinal items are rated according to a seven-point scale ranging from 0-6 with higher scores reflecting more severe eating disorder psychopathology. These items form four subscale scores including Restraint, Eating Concern, Shape Concern, and Weight Concern computed as the mean of constituent scale items. Discriminant validity, internal consistency, and concurrent validity are well documented for the EDE.

Brief Symptom Inventory-(BSI; Derogatis, 1993) The BSI is a shortened form of the revised version of the Symptom Checklist-90 (SCL-R-90; Derogatis, 1975, 1977), a selfreport measure of symptom levels reflecting psychopathology. This measure was employed to further characterize our sample given that affective comorbidities are common among those with AN. The BSI consists of 49-items that form nine symptom dimensions (i.e., Somatization, Obsession-Compulsion, Interpersonal Sensitivity, Depression, Anxiety, Hostility, Phobic Anxiety, Paranoid ideation, and Psychoticism). Participants indicate level of distress over the past seven days using a Likert scale ranging from " $o=$ Not at all" to " $4=$ Extremely." A Global Severity Index was derived by summing the dimensional scores with the four additional items and dividing by the total number completed items. Norms derived for adult females indicate a GSI mean of $0.35(S D=.37)$ for nonpatients $(n=358)$ and a mean of $1.40(S D=0.72)$ for psychiatric outpatients $(\mathrm{n}=577$; Derogatis, 1983). Depression means were $M=0.36(S D=0.56)$ for female nonpatients and $M=1.90(S D=1.05)$ for female psychiatric outpatients. Anxiety means were $M=0.44(S D=0.54)$ and $M=1.82$ ( $S D$ $=1.02$ ) for female nonpatients and female psychiatric outpatients respectively. The BSI has shown good internal consistency reliability for the nine dimensions with alpha coefficients ranging from 0.71 to 0.85 , and test-retest reliability coefficients ranging from 0.68 to 0.91 (Derogatis, 1993). Good convergent, construct, and predictive validity have been reported (Derogatis, 1993).

Body Shape Questionnaire-(BSQ; Cooper, Taylor, Cooper, \& Fairburn, 1987) The BSQ is a 34-item self-report measure designed to assess diverse aspects of body image disturbance including attitudes (e.g., dissatisfaction), cognitions (e.g. preoccupation with weight and shape), behaviors reflective of body image disturbance such as body checking or avoidance of public places due to body shame, and somatic experiences such as feelings of fatness (P. J. Cooper, et al., 1987). Items are scored on a 6-point scale from never to almostalways. Evidence of adequate internal consistency (.95-.97), test-retest reliability (.93), discriminant validity and construct validity have been established (P. J. Cooper, et al., 1987; Evans \& Dolan, 1993; Rosen, Jones, Ramirez, \& Waxman, 1996). We chose to include the BSQ, despite its overlap with the Shape/Weight Concerns subscales of the EDE and EDEQ, because of its inclusion of items that assess the experiential component of visceral sensitivity. That is, whereas there is overlap in the cognitive assessment of shape/weight concerns, the BSQ adds the additional assessment of disturbance in way the body is experienced, (e.g., Have you worried about your thighs spreading out when sitting down? Have you avoided running because your flesh might wobble?).

Temperament and Character Inventory-(Cloninger, Svrakic, \& Przybeck, 1993) The TCI is based on Cloniger's psychobiological model of personality development. Dimensions of temperament (e.g., Harm Avoidance, Reward Dependence, Novelty Seeking, Persistence) are purported to reflect biologically influenced behavioral response tendencies or more procedural-based learning. Character dimensions (e.g., Self-Directedness, Cooperativeness, Self-Transcendence) are purported to reflect environmentally influenced behavioral patterns, individual goals and values, or propositional-based learning. According to this framework, personality is a reflection of an individual's adaption to changing environmental contexts 
and thus knowledge of assets and deficits in both forms of learning contribute to a more complete understanding of human personality development (Cloninger, 1986; Cloninger, et al., 1993). Individuals with AN have been robustly characterized as elevated on harm avoidance relative to typically developing controls (Klump, et al., 2000), reflecting a longstanding transaction with the environment in which uncertainty (and thus potential for harm) is avoided. Use of this measure is intended to test the hypothesis that patterns of sensation may be associated with temperament.

\section{Statistical Method}

All data were visually inspected and relevant distribution parameters were calculated to guide selection of statistical procedures. All data were normally distributed and thus mixedeffect multivariate analysis of variance was employed to assess initial group differences on Sensory Profile subscales using group membership as the fixed effect and subscale scores on the Sensory Profile as the response variable. Linear regression analyses utilizing ordinary least-square criteria for model-fitting were computed. Examination of casewise diagnostics (standardized residuals, leverage, Dffit) was performed. Residuals $>2$ std. and leverage values $>(2$ (number of predictors $)+2 / n)$ were examined. No outliers were revealed using these parameters. Analyses were conducted using SPSS version 19 (IBM@SPSS Inc., () Chicago, IL). Based on the number of outcome variables, the family-wise corrected error rate is .008.

\section{Results \\ Demographic variables}

Demographic and clinical features of the sample are presented in Table 1. Participants were primarily Caucasian (81.3\%), single (85\%), educated (63.8\% had 16 or more years of education), and intelligent - with an intelligence quotient more than 1.5 standard deviations above the population mean. Groups did not differ on age, race, or IQ. By design, groups differed on BMI: those with AN had a significantly lower BMI than AN-WR or CN, while AN-WR was not different from CN (Table 2).

\section{Sensory Profile Group Comparisons}

Using a multivariate model with group as a fixed between-subject effect, the combined multivariate mean on the four subscales of the SP was significant $(p<.005)$, with subsequent tests of between subjects effects revealing group differences on Sensory Sensitivity $\left(F=9.29, p<.001, \eta_{\mathrm{p}}{ }^{2}=.26\right)$ and Sensory Avoidance $\left(F=7.16, p<.002, \eta_{\mathrm{P}}{ }^{2}=\right.$. 21). As shown in Table 3, post-hoc comparisons indicated that those with AN reported greater sensitivity to sensation than $\mathrm{CN}$ and were more likely to actively avoid sensations than CN. The same pattern of results was indexed in the AN-WR group (Figure 2): the weight-restored group reported greater sensitivity to sensation and more likely to avoid sensation than the control group. The two clinical groups did not differ from each other on these measures, suggesting that susceptibility to AN and not weight per se was the relevant factor.

\section{Sensation and Eating Disorder Variables}

Sensory Sensitivity was negatively and significantly correlated with lowest BMI $\left(r^{2}=-.32\right.$, $p<.001)$, but not current BMI $\left(r^{2}=.03, p=.18\right)$, again supporting a link to AN susceptibility rather than weight. This relationship between Sensory Sensitivity and past weight history was paralleled by a relationship between Sensory Sensitivity and several subjective measures relating to the experience of the body. Zero-order correlations between the BSQ and Sensory Sensitivity were significant, $r(56)=.51, p<.0001$, indicating the body 
image disturbance increased with increased global sensitivity to sensation. We conducted a moderated regression analysis to examine whether the relationship between sensory sensitivity and body image disturbance (the response variable) differed as a function of group membership. Predictor variables were centered prior to analysis. While the overall model was significant, $\mathrm{R}^{2}=.67, F=20.9, p<.001$, the interaction term was not significant. In other words, the relationship between sensory sensitivity and body image disturbance, when present, was comparable in all three groups. As a further exploratory probe of the nature of sensory sensitivity on the experience of the body, we examined the relationship of sensory sensitivity to experiential aspects of body image disturbance: somatic preoccupation, feelings of fatness relative to cognitive aspects: fear of stopping eating based on items from the EDE-Q. Sensory sensitivity was significantly correlated with the feeling of fatness: $r=.55, p<.001$ and somatic preoccupation, $r=.45, p<.001$, but not fears of overeating: $r=.32 p<.02$.

\section{Sensation and Temperament}

Finally, the association of temperament harm avoidance with sensitivity to sensation was highly significant $r=.61, p<.0001$. We conducted a moderated regression analysis to examine whether the relationship between sensory sensitivity with harm avoidance (the response variable) differed as a function of group membership (Figure 1). The overall model was significant, $\mathrm{R}^{2}=.45, F=8.29, p<.001$. The simple effect of self-perceived sensory sensitivity on harm avoidance was significant, although only at a trend level, $\beta=.31, p=$. 04 . However the interaction term, indexing moderation, was not significant indicating that the relationship of sensory sensitivity to harm avoidance does not differ as a function of clinical status.

\section{Exploratory Analyses}

Finally, to better understand the nature of somatic experience, we examined sensitivity to the specific sensorium that may distinguish groups. Given our limited sample size and the exploratory nature of this work, we limited examination to those senses that have the greatest face validity for somatosensory influences on body image disturbance: sensitivity to touch, vision, and movement. We standardized these scores so relative deviations on these sensory domains could be visually inspected. The overall multivariate test was significant, Wilk's $£(6)=4.31, p<.001$, with resulting between group comparisons revealing differences across all measures (Figure 2).

\section{Discussion}

\section{Sensitization and Body Image Disturbance}

This study provides novel evidence that, in addition to exhibiting cognitive components of body image disturbance, individuals with AN report difficulties in the subjective experience of bodily sensations. Specifically, we found that women with AN, whether currently ill or restored to a healthy BMI, reported enhanced subjective sensitivity to sensory experience (e.g., taste, touch, vision) and increased attempts to avoid sensory experience, relative to control women. Furthermore, such sensitivity was positively correlated with cognitive aspects of body image disturbance such as the negative evaluation of appearance and negative interpretations of visceral experience (e.g., feeling fat). Taken together, these findings suggest that both enhanced sensitization and aberrant conditioning may provide useful heuristics to understand the nature of body image disturbance in AN.

Awareness of sensation and/or sensitivity to sensation does not necessarily lead to negatively valenced interpretations of body experience or avoidance of bodily states. Sensitivity may, however, provide a context in which the aberrant evaluation of physical 
sensation is more likely to occur thus resulting in subsequent avoidance. One possible pathway is via social learning. For example, sensitivity to sensation may enhance awareness of the physiological correlates of emotional experience (e.g., the churning in the gut that may accompany anxiety). Depending on an individual's learning history and accurate "social biofeedback" (Buck, 1999; Skinner, 1945), an individual may learn adaptive or maladaptive interpretations of the meaning of these visceral sensations. For example, when a child notices a churning sensation in her gut, she may interpret it as hunger, anxiety, or a signal of weight gain or fatness, etc. These interpretations depend on the complex developmental interplay of an individual with his/her social environment. Individuals in the social environment model responsiveness to their own interoceptive states and attach labels to and respond to the child's state and thereby help a child learn to decipher his/her interoceptive milieu. Over time, sensations can become inextricably associated with the verbal description used to discriminate that sensation. If this verbal label is itself associated with an aversive learning history, then the experience of that sensation will be avoided in an effort to avoid its associated verbally-mediated aversive connotations (e.g., if a churning sensation in the gut has co-occured with the interpretation that one "is fat" and fat is aversive then this associated sensation is avoided at all costs (Mesulam, 1998; Widen \& Russell, 2008). Over time, the nuanced and diverse meanings of somatic sensations, meanings that vary according to context, will be diminished as the over-generalized verbal interpretation guides subsequent behavior rather than somatic experience (e.g., if a churning sensation in the gut has co-occurred with the interpretation that one "is fat" then this sensation comes to signal "fatness" regardless of context). Thus, individuals with AN may attempt to avoid sensory experience because of negatively valenced interpretations developed through aberrant conditioning.

A second possible pathway is via the association of stimulus intensity with discomfort, pain, or aversion (Carretie, Mercado, Tapia, \& Hinojosa, 2001). There is evidence that stimulus features and the valence placed on those features are reciprocally influential (e.g., young children cry in response to very loud noises and the aversiveness experienced in response to a stimulus can alter perceptual thresholds)(Schechtman, Laufer, \& Paz, 2010). Self-reported sensitivity as indexed in this study does not provide evidence that individuals with AN are objectively more sensitive than their healthy control counterparts (e.g., evidence lower detection or discrimination thresholds to sensory manipulations). Nevertheless, current findings suggest the importance of future research in this arena as evidence of objective sensitivity to sensation in AN would provide a critical counter-thesis to current hypotheses about the nature of body image disturbance. In particular, interoceptive deficits in AN may not be a result of hyposensitivity as has been historically described (Bruch, 1962). Rather, individuals with AN may instead be hypersensitive to sensory experience which would suggest alternative pathways to deficits in interoceptive awareness and disturbance in the way the body is experienced.

For example, sensitivity to sensation, aberrant conditioning, and learned avoidance may combine to contribute to body image disturbance. That is if physical sensations are experienced as aversive, then first, avoidance of such sensations would be negatively reinforced. Second, avoidance may generalize to less noxious sensations. Third, the continued negative reinforcement provided by the avoidance of physical sensation would prevent the opportunity for adaptive interpretations to develop. Fourth, given that the meaning of visceral sensations becomes increasingly elaborated throughout development (e.g., the stomach pains of childhood evolve into the butterflies of anxiety), the elaboration of visceral states may fail to develop given the early onset of AN. For example, the avoidance of sensations associated with touch (such as the feeling of fabric on the skin), may generalize to the avoidance of sensations that stretch skin (e.g. fullness in the gut). Avoidance interferes with the habituation of the experience and prevents alternative ways of 
relating, interpreting, and responding to the physical sensation from developing. While laboratory studies are needed to clarify these potential pathways linking interoceptive sensitivity and the accurate elaboration of visceral states to body image disturbance, findings from this study suggest a novel theoretical framework from which to examine the development and maintenance of AN symptoms.

\section{Sensitization and BMI}

We propose three alternative hypothetical interpretations to explain the association of lowest lifetime BMI with sensory sensitivity, all of which would require further research to refute or support. First, this association may be a reflection of illness severity. Lowest illnessrelated BMI has been reported in several investigations to be a marker of illness severity (Dechartres et al., 2011; Hofman, Landewe-Cleuren, Wojciechowski, \& Kruseman, 2009; Mehler, Sabel, Watson, \& Andersen, 2008). As such, lowest BMI may be associated with more extreme endorsement of all symptoms - including sensitivity to sensation and avoidance of sensation. Second, attaining a dangerously low BMI may be an attempt to mute sensory experience. Thus, examination of the manner in which somatic experience is altered in the starved state may provide important clues as to why those with AN will work to maintain this state. As demonstrated in Figure 2, those currently diagnosed with AN differed from those who were weight-restored individuals with respect to specific aspects of sensory sensitivity. Specifically individuals currently ill with AN were more sensitive to touch while those who were weight-restored were more sensitive to movement. Further research is needed to understand the subjective valence placed on these specific alterations in experience, which may inform the functional nature of symptom development. Notably, those particularly sensitive to sensation may find such muting to be particularly reinforcing. Third, it is conceivable that a dangerously low BMI can impact sensory thresholds. Given the severe threat to survival associated with a dangerously low BMI, it would not be surprising if this severely deprived visceral state heightened vigilance to sensation (e.g. vision, sound) to facilitate escape of a vulnerable organism. In partial support of these findings. Goldzak et al. (2012) document enhanced auditory acuity and kinesthesia in a small sample of adolescents with AN relative to healthy controls (Goldzak-Kunik, et al., 2012). Combined, such data highlight the need for further research to study the impact of BMI itself on body image disturbance or the influence of sensation on body image development. While data from the current study cannot distinguish between these hypotheses, we offer these to help guide further inquiry.

\section{Sensitization and Temperament}

Developmental researchers have long recognized the critical role of sensory sensitivity in temperament (Ben-Sasson, et al., 2009; Goldsmith, et al., 2006). Thus, it is not surprising that findings from the current study support the association between sensitivity to sensation and harm avoidance. Certainly not all individuals with high harm avoidance develop AN. However, sensory sensitivity may be a precursor or setting condition for the avoidance of aversive sensory experiences. Moreover, when aberrant conditioning promotes the negative association of bodily experience, cognitive symptoms of AN may develop (e.g., excessive concern with weight and shape) and behavioral symptoms may emerge as a means to attenuate such negative bodily states (e.g., starvation). In this way, sensitization and harm avoidance may promote aberrant conditioning that fosters the development of body image disturbance.

\section{Implications for Clinical Practice}

Additional empirical confirmation of enhanced sensitization would provide a theoretical frame from which to inform treatment interventions for body image disturbance. One of the most empirically-supported strategies for the management of body image disturbance is 
mirror exposure (Delinsky \& Wilson, 2006). This intervention targets over concern with body size and shape and body dissatisfaction by using graduated exposure to one's visual image. During graduated exposure to distressing body parts, various cognitive approaches have been employed: mindfulness, nonjudgmental descriptions, and activation of cognitive dissonance. In all cases, these approaches were attempts to alter aversive conditioning of an individual's image and subsequent distressing thoughts and feelings by pairing an individual's visual image with either neutral (e.g., in the cause of the nonjudgmental condition) or positive statements (e.g., in the case of the cognitive dissonance condition). Critically, all three cognitive and behavioral strategies were intended to alter attitudes and experiences related to visual body perception.

Results from the current study suggest that clinical practice may potentially be improved by expanding the targets of exposure to include sensory experiences beyond the visual realm (i.e., including internal sensations) much in the same way that treatment for anxiety disorders has expanded to include interoceptive cues associated with anxiety (e.g., racing heart). For instance, graduated exposure to tighter or looser clothing, causing gut stimulation via vestibular provocations or mood inductions, and other sensory exposures may serve as necessary additions to the treatment of body image disturbance.

Additionally, if enhanced sensitization and somatic preoccupation are indeed persistent, biologically-influenced features that are slow to change, exposure to bodily sensation may be further enhanced by acceptance-based strategies. Strategies that recontextualize sensation and focus on changing how one relates and responds to physical sensations may be more effective than trying to change the experiences themselves. As such, rather than teaching ways to attenuate or down-regulate sensation (which may prove futile), embedding sensation in the context of a broader emotional experience might decrease negative reactivity and facilitate more effective responding to these sensations. Enhanced sensory experience could be also be re-contextualized as a tool for living a valued, vital life. Indeed, arousability allows us to ascertain what activities are meaningful to pursue (as well as avoid), and experiencing sensation as a signal that one is engaging activities that are life building could allow individuals to relinquish unhelpful attempts to quell such sensation. Additional research is needed to determine whether these theoretically coherent and novel treatment directions are effective management strategies for the treatment of body image disturbance.

\section{Limitations}

The primary limitations of the study are the small sample size and use of self-report measures, rather than laboratory studies, to describe sensory experience. While the strength of this strategy is that is permits the measurement of perception of sensory experience, it precludes the objective measurement of sensory experience. Further, among our clinical group, we employed a group that was weight-restored rather than a purely recovered group. This design choice could be construed as a strength or a weakness. As a strength, we were able to examine the effects of weight independent of the cognitive improvement that may arise with full recovery. As the boundaries of cognitive change that delineate recovery in AN remain ill-defined, this could be considered a conservative strategy. However, future studies would benefit both from psychophysical testing in addition to self-report as well as the use of a more robustly recovered group. Finally, our experimental groups were racially and ethnically homogenous which limits the generalizability of study findings.

\section{Summary}

In AN, persistent awareness of the body impinges on ongoing function. While the cognitions that accompany AN are frequently described as ego-syntonic,(Sunday, Halmi, \& Einhorn, 1995) physical sensations are not, which distinguishes the experience of the body from the 
negatively valenced cognitions about the body. Study of subjective sensitivity to sensation and its relationship to body image disturbance has been a limited focus of research in AN. This is a preliminary pilot study based solely on self-report measures. Yet, the strength of the associations highlights the potential of this area of study to further elucidate the nature of body image disturbance in AN.

\section{Acknowledgments}

This work was supported by the NIMH and NIDDK grants awarded to Dr. Zucker (R01-MH-078211-01, RC1MH-088678, K23-MH-070418).

This research is supported by NIH grants R01-MH-078211, K23-MH-070418 (Zucker).

\section{References}

American Psychiatric Association. Diagnostic and Statistical Manual for Psychiatric Disorders. 4th. Washington, DC: American Psychiatric Press; 1994.

Anema HA, van Zandvoort MJE, de Haan EHF, Kappelle LJ, de Kort PLM, Jansen BPW, Dijkerman HC. A double dissociation between somatosensory processing for perception and action. Neuropsychologia. 2009; 47(6):1615-1620.10.1016/j.neuropsychologia.2008.11.001 [PubMed: 19038277]

Beauchaine TP, Gatzke-Kopp L, Mead HK. Polyvagal Theory and developmental psychopathology: Emotion dysregulation and conduct problems from preschool to adolescence. Biological Psychology. 2007; 74(2):174-184. [PubMed: 17045726]

Ben-Sasson A, Carter AS, Briggs-Gowan MJ. Sensory Over-Responsivity in Elementary School: Prevalence and Social-Emotional Correlates. Journal of Abnormal Child Psychology. 2009; 37(5): 705-716.10.1007/s10802-008-9295-8 [PubMed: 19153827]

Binford RB, Le Grange D, Jellar CC. Eating Disorders Examination Versus Eating Disorders Examination-Questionnaire in Adolescents with Full and Partial-Syndrome Bulimia Nervosa and Anorexia Nervosa. International Journal of Eating Disorders. 2005 Jan; 2005 37(1):44-49. [PubMed: 15690465]

Bruch H. Perceptual and conceptual disturbances in anorexia nervosa. Psychosomatic Medicine. 1962; 24(2):187-194. [PubMed: 13873828]

Buck R. The biological affects: A typology. Psychological Review. 1999; 106(2):301-336. [PubMed: 10378015]

Carretie L, Mercado F, Tapia M, Hinojosa JA. Emotion, attention, and the 'negativity bias', studied through event-related potentials. International Journal of Psychophysiology. 2001; 41(1):7585.10.1016/s0167-8760(00)00195-1 [PubMed: 11239699]

Chen YH, Rodgers J, McConachie H. Restricted and Repetitive Behaviours, Sensory Processing and Cognitive Style in Children with Autism Spectrum Disorders. Journal of Autism and Developmental Disorders. 2009; 39(4):635-642.10.1007/s10803-008-0663-6 [PubMed: 19015969]

Cloninger CR. A unified biosocial theory of personality and its role in the development of anxiety states. Psychiatric Developments. 1986; 3:167-226. [PubMed: 3809156]

Cloninger CR, Svrakic DM, Przybeck TR. A Psychobiological Model of Temperament and Character. Archives of General Psychiatry. 1993; 50(12):975-990. [PubMed: 8250684]

Colbert HA, Bargmann CI. Environmental signals modulate olfactory acuity, discrimination, and memory in Caenorhabditis elegans. Learning \& Memory. 1997; 4(2):179-191.10.1101/1m.4.2.179 [PubMed: 10456062]

Cooper PJ, Taylor MJ, Cooper Z, Fairburn CG. The development and validation of the Body Shape Questionnaire. International Journal of Eating Disorders. 1987; 6:485-494.

Cooper Z, Fairburn CG. The Eating Disorder Examination: A semi-structured interview for the assessment of the specific psychopathology of eating disorders. International Journal of Eating Disorders. 1987; 6(1):1-8. 
Cserjesi R, Vermeulen N, Luminet O, Marechal C, Nef F, Simon Y, Lenard L. Explicit vs. implicit body image evaluation in restrictive anorexia nervosa. Psychiatry Research. 2010; 175(1-2):148153.10.1016/j.psychres.2009.07.002 [PubMed: 19931183]

Dechartres A, Huas C, Godart N, Pousset M, Pham A, Divac SM, Falissard B. Outcomes of Empirical Eating Disorder Phenotypes in a Clinical Female Sample: Results from a Latent Class Analysis. Psychopathology. 2011; 44(1):12-20.10.1159/000315362 [PubMed: 20980783]

Dei M, Seravalli V, Bruni V, Balzi D, Pasqua A. Predictors of recovery of ovarian function after weight gain in subjects with amenorrhea related to restrictive eating disorders. Gynecological Endocrinology. 2008; 24(8):459-464.10.1080/09513590802246141 [PubMed: 18850384]

Delinsky SS, Wilson GT. Mirror exposure for the treatment of body image disturbance. International Journal of Eating Disorders. 2006; 39(2):108-116. [PubMed: 16231342]

Derogatis, LR. SCL-90-R administration, scoring, and procedures manual. Baltimore, MD: Clinical Psychometric Research; 1983.

Derogatis, LR. BSI Brief Symptom Inventory Administration, Scoring, and Procedures Manual. 4th. Minneapolis, MN: National Computer Systems; 1993.

Dunn, W. Sensory Profile User's Manual. San Antonio, TX: Harcourt Assessment; 1999.

Evans C, Dolan B. Body Shape Questionnaire - Derivation of Shortened Alternate Forms. International Journal of Eating Disorders. 1993; 13(3):315-321.10.1002/1098-108x(199304)13:3<315: :aideat2260130310>3.0.co;2-3 [PubMed: 8477304]

Fairburn CG, Beglin SJ. The assessment of eating disorders: interview or self-report questionnaire? International Journal of Eating Disorders. 1994; 16:363-370. [PubMed: 7866415]

Fairburn, CG.; Cooper, Z. The Eating Disorder Examination. 12th. New York: Guilford Press; 1993.

Fairburn CG, Stice E, Cooper Z, Doll HA, Norman PA, O'Connor ME. Understanding persistence in bulimia nervosa: a 5-year naturalistic study. Journal of Consulting and Clinical Psychology. 2003; 71:103-109. [PubMed: 12602430]

Fecteau JH, Munoz DP. Salience, relevance, and firing: A priority map for target selction. Trends in Cognitive Sciences. 2006; 10(8):382-390. [PubMed: 16843702]

Fichter MM, Elton M, Engel K, Meyer A, Mall H, Poustka F. Structured interview for anorexia and bulimia nervosa (SIAB): Development of a new instrument for the assessment of eating disorders. International Journal of Eating Disorders. 1991; 10(5):571-592.

Fisher S, Cleveland SE, Davis A. Prediction of Physioloigcal Reactivity from a Body-Image Schema. American Psychologist. 1957; 12(4):426-426.

Gabriels RL, Agnew JA, Miller LJ, Gralla J, Pan Z, Goldson E, Hooks E. Is there a relationship between restricted, repetitive, stereotyped behaviors and interests and abnormal sensory response in children with autism spectrum disorders? Research in Autism Spectrum Disorders. 2008; 2(4): 660-670.10.1016/j.rasd.2008.02.002

Gallagher S, Cole J. Body image and body schema in a deafferented subject. Journal of Mind and Behavior. 1995; 16(4):369-389.

Garner, DM. Measurement of eating disorder psychopathology. In: B, KD.; Fairburn, CG., editors. Eating disorders and obesity: A comprehensive handbook. 2nd. New York: Guilford Press; 2002. p. 141-146.

Gleaves DH, Williamson DA, Eberenz KP, Sebastian SB, Barker SE. Clarifying body-image disturbance: Analysis of a multidimensional model using structural modelling. Journal of Personality Assessment. 1995; 64:478-493. [PubMed: 16367723]

Goldsmith HH, Van Hulle CA, Arneson CL, Schreiber JE, Gernsbacher MA. A population-based twin study of parentally reported tactile and auditory defensiveness in young children. Journal of Abnormal Child Psychology. 2006; 34(3):393-407.10.1007/s10802-006-9024-0 [PubMed: 16649001]

Goldzak-Kunik G, Friedman R, Spitz M, Sandler L, Leshem M. Intact sensory function in anorexia nervosa. American Journal of Clinical Nutrition. 2012; 95(2):272-282.10.3945/ajen.111.020131 [PubMed: 22205316]

Gray JA. Psychophysiological Basis of Introversion-Extraversion. Behaviour Research and Therapy. 1970; 8(3):249-\&. [PubMed: 5470377]

Guimon J. From the body schema to the body image. 1997 
Halmi KA, Sunday SR. Temporal patterns of hunger and fullness ratings and related cognitions in anorexia and bulimia. Appetite. 1991; 16(3):219-237. [PubMed: 1883249]

Hofman M, Landewe-Cleuren S, Wojciechowski F, Kruseman AN. Prevalence and clinical determinants of low bone mineral density in anorexia nervosa. European Journal of Internal Medicine. 2009; 20(1):80-84.10.1016/j.ejim.2008.04.016 [PubMed: 19237098]

Jacobi C, Hayward C, de Zwaan M, Kraemer HC, Agras WS. Coming to terms with risk factors for eating disorders: Application of risk terminology and suggestions for a general taxonomy. Psychological Bulletin. 2004; 130:19-65. [PubMed: 14717649]

Kammers MPM, de Vignemont F, Verhagen L, Dijkerman HC. The rubber hand illusion in action. Neuropsychologia. 2009; 47(1):204-211.10.1016/j.neuropsychologia.2008.07.028 [PubMed: 18762203]

Keel PK, Dorer DJ, Franko DL, Jackson SC, Herzog DB. Postremission predictors of relapse in women with eating disorders. American Journal of Psychiatry. 2005; 162(12):2263-2268. [PubMed: 16330589]

Keizer A, Smeets MAM, Dijkerman HC, van den Hout M, Klugkist I, van Elburg A, Postma A. Tactile body image disturbance in anorexia nervosa. Psychiatry Research. 2011; 190(1):115-120.10.1016/ j.psychres.2011.04.031 [PubMed: 21621275]

Kern JK, Garver CR, Carmody T, Andrews AA, Trivedi MH, Mehta JA. Examining sensory quadrants in autism. Research in Autism Spectrum Disorders. 2007; 1(2):185-193.10.1016/j.rasd. 2006.09.002

Killen JD, Taylor CB, Hayward C, Wilson DM, Haydel KF, Hammer LD, Kraemer HC, et al. Pursuit of thinness and onset of eating disorder symptoms in a comunity sample of adolescent girls: a three-year prospective analysis. International Journal of Eating Disorders. 1994; 16:227-238. [PubMed: 7833956]

Klump KL, Bulik CM, Pollice C, Halmi KA, Fichter MM, Berrettini WH, Kaye WH. Temperament and character in women with anorexia nervosa. Journal of Nervous \& Mental Disease. 2000; 188(9):559-567. [PubMed: 11009328]

Klump KL, Strober M, Bulik CM, Thornton L, Johnson C, Devlin B, Kaye WH. Personality characteristics of women before and after recovery from an eating disorder. Psychological Medicine. 2004; 34(8):1407-1418. [PubMed: 15724872]

Korbonits M, Blaine D, Elia M, Powell-Tuck J. Metabolic and hormonal changes during the refeeding period of prolonged fasting. European Journal of Endocrinology. 2007; 157(2):157-166.10.1530/ eje-06-0740 [PubMed: 17656593]

Laycock R, Crewther SG, Crewther DP. A role for the 'magnocellular advantage' in visual impairments in neuro developmental and psychiatric disorders. Neuroscience and Biobehavioral Reviews. 2007; 31(3):363-376.10.1016/j.neubiorev.2006.10.003 [PubMed: 17141311]

LeBoeuf B, Guo X, Garcia LR. The effects of transient starvation persist through direct interactions between CaMKII and ether-a-go-go K+ channels in C. elegans males. Neuroscience. 2011; 175:117.10.1016/j.neuroscience.2010.12.002 [PubMed: 21145946]

Luce KH, Crowther JH. The reliability of the Eating Disorder Examination-Self-Report Questionnaire Version (EDE-Q). International Journal of Eating Disorders. 1999; 25(3):349-351. [PubMed: 10192002]

Martucci KT, Yelle MD, Coghill RC. Differential effects of experimental central sensitization on the time-course and magnitude of offset analgesia. Pain. 2012; 153(2):463-472.10.1016/j.pain. 2011.11.010 [PubMed: 22154333]

McEwen BS. Protection and damage from acute and chronic stress - Allostasis and allostatic overload and relevance to the pathophysiology of psychiatric disorders. Biobehavioral Stress Response: Protective and Damaging Effects. 2004:1-7.

Mehler PS, Sabel AL, Watson T, Andersen AE. High Risk of Osteoporosis in Male Patients with Eating Disorders. International Journal of Eating Disorders. 2008; 41(7):666-672.10.1002/eat. 20554 [PubMed: 18528874]

Mesulam MM. From sensation to cognition. Brain. 1998; 121:1013-1052.10.1093/brain/121.6.1013 [PubMed: 9648540] 
Misra M, Prabhakaran R, Miller KK, Tsai P, Lin A, Lee N, Klibanski A. Role of cortisol in menstrual recovery in adolescent girls with anorexia nervosa. Pediatric Research. 2006; 59(4):598_ 603.10.1203/01.pdr.0000203097.64918.63 [PubMed: 16549537]

Moehler E, Kagan J, Parzer P, Wiebel A, Brunner R, Resch F. Relation of behavioral inhibition to neonatal and infant cardiac activity, reactivity and habituation. Personality and Individual Differences. 2006; 41(7):1349-1358.10.1016/j.paid.2006.05.008

Mohr HM, Zimmermann J, Roder C, Lenz C, Overbeck G, Grabhorn R. Separating two components of body image in anorexia nervosa using fMRI. Psychological Medicine. 2010; 40(9):15191529.10.1017/s0033291709991826 [PubMed: 19917143]

Mond JM, Hay PJ, Rodgers B, Owen C, Beumont PJV. Temporal stability of the eating disorder examination questionnaire. International Journal of Eating Disorders. 2004; 36(2):195-203. [PubMed: 15282689]

Nickel FT, Seifert F, Lanz S, Maihofner C. Mechanisms of neuropathic pain. European Neuropsychopharmacology. 2012; 22(2):81-91.10.1016/j.euroneuro.2011.05.005 [PubMed: 21672666]

Park RJ, Dunn BD, Barnard PJ. Schematic Models and Modes of Mind in Anorexia Nervosa I: A Novel Process Account. International Journal of Cognitive Therapy. 2011; 4(4):415-437.

Peterson CB, Crosby RD, Wonderlich SA, Joiner T, Crow SJ, Mitchell JE, le Grange D. Psychometric properties of the eating disorder examination-questionnaire: Factor structure and internal consistency. International Journal of Eating Disorders. 2007 May; 2007 40(4):386-389. [PubMed: 17304585]

Pfeiffer B, Kinnealey M, Reed C, Herzberg G. Sensory modulation and affective disorders in children and adolescents with Asperger's disorder. American Journal of Occupational Therapy. 2005; 59(3):335-345. [PubMed: 15969281]

Radomsky AS, de Silva P, Todd G, Treasure J, Murphy T. Thought-shape fusion in anorexia nervosa: an experimental investigation. Behaviour Research \& Therapy. 2002; 40(10):1169-1177. [PubMed: 12375725]

Rimm-Kaufman SE, Kagan J. Infant predictors of kindergarten behavior: The contribution of inhibited and uninhibited temperament types. Behavioral Disorders. 2005; 30(4):331-347.

Roberto CA, Steinglass J, Mayer LE, Attia E, Walsh BT. The clinical significance of amenorrhea as a diagnostic criterion for anorexia nervosa. Int J Eat Disord. 2008; 41(6):559-563. [PubMed: 18454485]

Rosen JC, Jones A, Ramirez E, Waxman S. Body shape questionnaire: Studies of validity and reliability. International Journal of Eating Disorders. 1996; 20(3):315-319.10.1002/ (sici)1098-108x(199611)20:3<315: :aid-eat11>3.0.co;2-z [PubMed: 8912044]

Sachdev P, Mondraty N, Wen W, Gulliford K. Brains of anorexia nervosa patients process self-images differently from non-self-images: An fMRI study. Neuropsychologia. 2008; 46(8):21612168.10.1016/j.neuropsychologia.2008.02.031 [PubMed: 18406432]

Schechtman E, Laufer O, Paz R. Negative Valence Widens Generalization of Learning. Journal of Neuroscience. 2010; 30(31):10460-10464.10.1523/jneurosci.2377-10.2010 [PubMed: 20685988]

Seeger G, Braus DF, Ruf M, Goldberger U, Schmidt MH. Body image distortion reveals amygdala activation in patients with anorexia nervosa -- a functional magnetic resonance imaging study. Neurosci Lett. 2002; 326(1):25-28. [PubMed: 12052530]

Shafran R, Robinson P. Thought-shape fusion in eating disorders. British Journal of Clinical Psychology. 2004; 43:399-408. [PubMed: 15530210]

Skinner BF. The operational analysis of psychological terms. Psychological Review. 1945; 52:270277.

Sunday SR, Halmi KA, Einhorn A. The Yale-Brown-Cornell Eating Disorder Scale - a New Scale to Assess Eating Disorder Symptomatology. International Journal of Eating Disorders. 1995; 18(3): 237-245. [PubMed: 8556019]

Thompson RF. Habituation: A history. Neurobiology of Learning and Memory. 2009; 92(2):127134.10.1016/j.nlm.2008.07.011 [PubMed: 18703156]

Vierck CJ, Riley JL, Wong F, King CD, Mauderli AP. Psychophysical demonstration of bidirectional pain modulation (sensitization and desensitization) by ascending or descending progressions of 
thermal stimulus intensity. Brain Research. 2010; 1347:58-64.10.1016/j.brainres.2010.06.006 [PubMed: 20537988]

Vocks S, Busch M, Gronemeyer D, Schulte D, Herpertz S, Suchan B. Differential neuronal responses to the self and others in the extrastriate body area and the fusiform body area. Cognitive Affective \& Behavioral Neuroscience. 2010; 10(3):422-429.10.3758/cabn.10.3.422

Wang T, Hung CCY, Randall DJ. The comparative physiology of food deprivation: From feast to famine. Annual Review of Physiology. 2006; 68:223-251.10.1146/annurev.physiol. 68.040104.105739

Watanabe M. Functional relationships between body image and body schema in motor action. Journal of Sport \& Exercise Psychology. 2005; 27:S157-S157.

Wechsler, D. WASI: Wechsler Abbreviated Scale of Intelligence. San Antonio, TX: The Psychological Corporation; 1999.

Widen SC, Russell JA. Children acquire emotion categories gradually. Cognitive Development. 2008; 23(2):291-312.10.1016/j.cogdev.2008.01.002 


\section{Highlights}

- Examined the relationship of subjective sensory sensitivity to body image disturbance in adults with anorexia nervosa

- Currently ill and weight-restored women with AN reported enhanced subjective sensitivity

- AN groups also reported increased attempts to avoid sensory experience

- Subjective sensitivity significantly positively correlated with body image disturbance

- Subjective sensitivity significantly positively correlated with harm avoidance

- Subjective sensitivity was associated with lowest lifetime BMI but not current BMI 


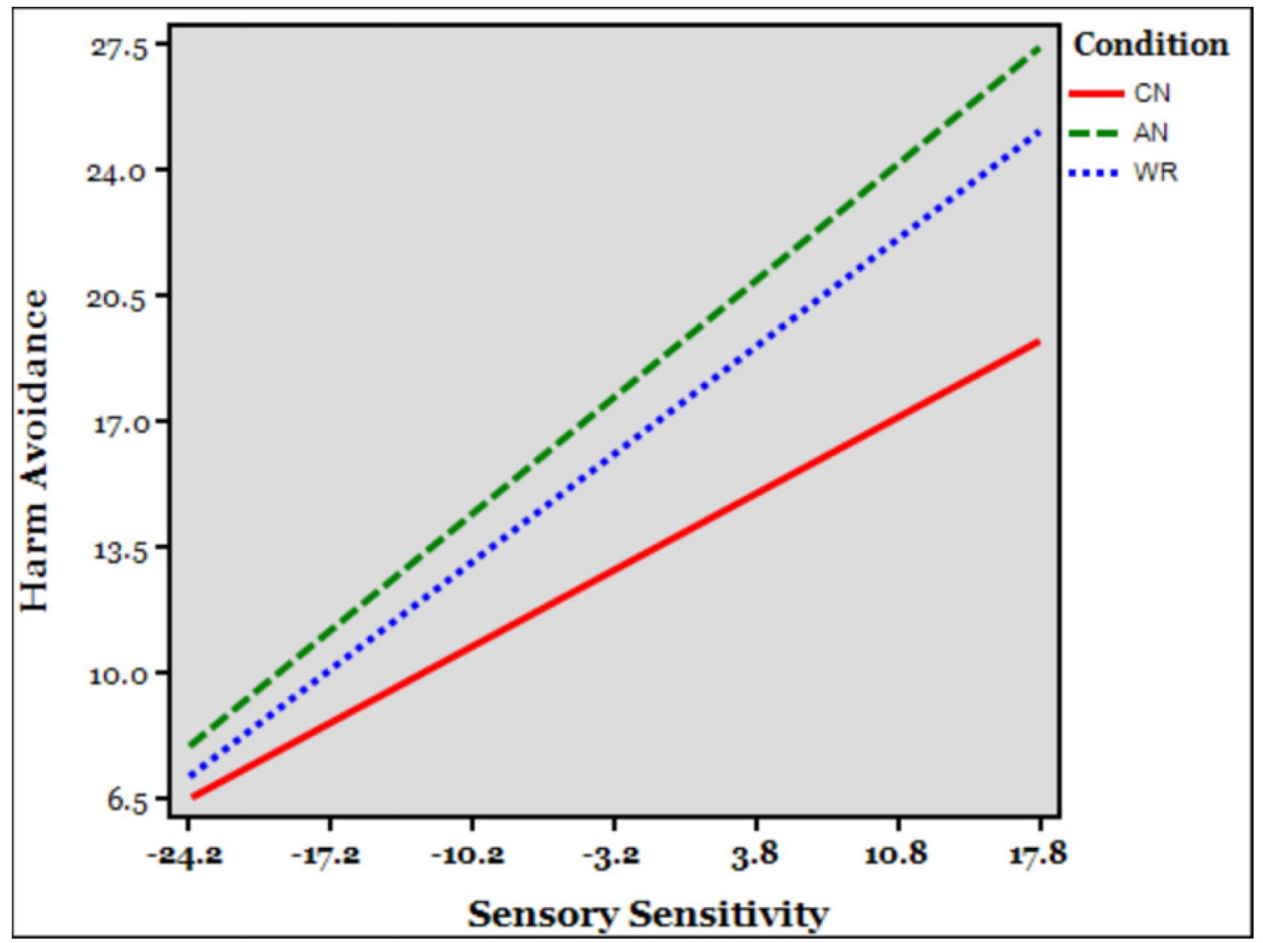

Figure 1.

Visual display of moderated regression analysis examining the relationship of sensory sensitivity to harm avoidance as a function of group membership. The relationship was positive and linear with simple slopes significant across all groups, $\mathrm{p}<.01$. $\mathrm{CN}=$ controls, $\mathrm{AN}=$ current $\mathrm{AN}, \mathrm{WR}=$ weight restored with history of $\mathrm{AN}$. 


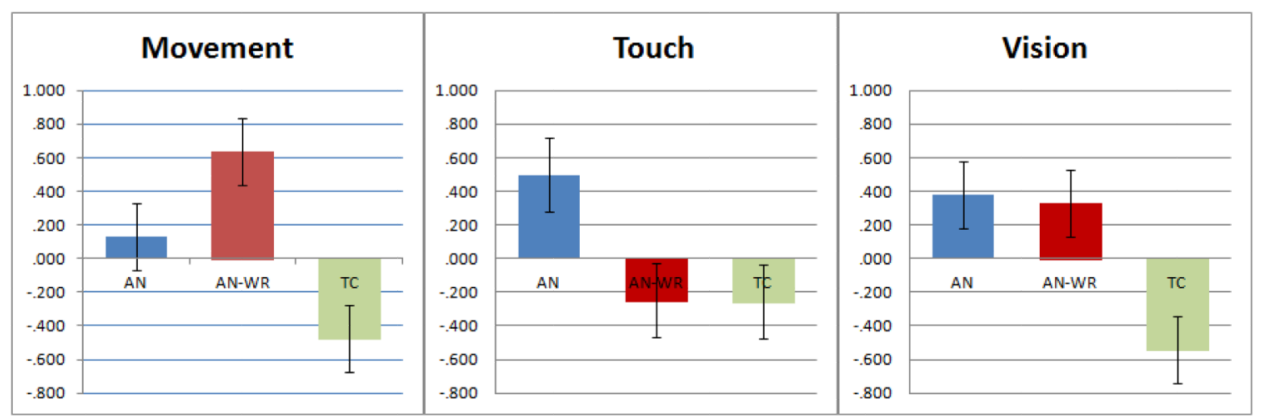

Figure 2.

A plot of standardized scores of sensory dimensions across groups. The clinical and weight restored groups demonstrated unique patterns of sensitivity. 


\section{Demographic Information}

Table 1

\begin{tabular}{|c|c|c|c|c|}
\hline Demographic Variable & AN $(n=20)$ & WR $(n=15)$ & $\mathrm{CN}(n=24)$ & Total $(n=59)$ \\
\hline \multicolumn{5}{|l|}{ Age } \\
\hline Mean (SD) & $25.6(8.7)$ & $27.4(10.1)$ & $26.6(9.8)$ & $27.2(9.4)$ \\
\hline $18-25$ & $8(38.1 \%)$ & $12(60 \%)$ & $14(60 \%)$ & $34(53.1 \%)$ \\
\hline $26-30$ & $8(38.1 \%)$ & $2(10 \%)$ & $3(13 \%)$ & $13(20.3 \%)$ \\
\hline $31-35$ & $1(4.8 \%)$ & $2(10 \%)$ & $3(13 \%)$ & $6(9.4 \%)$ \\
\hline $36-40$ & $2(9.5 \%)$ & $2(10 \%)$ & $1(4.3 \%)$ & $5(7.8 \%)$ \\
\hline $41-45$ & $1(4.8 \%)$ & $1(5 \%)$ & $0(0 \%)$ & $2(3.1 \%)$ \\
\hline Over 45 & $1(4.8 \%)$ & $1(5 \%)$ & $2(8.7 \%)$ & $4(6.3 \%)$ \\
\hline \multicolumn{5}{|l|}{ Race/Ethnicity } \\
\hline White/Caucasian & $21(100 \%)$ & $18(90 \%)$ & $13(56.5 \%)$ & $52(81.3 \%)$ \\
\hline Black/African American & $0(0 \%)$ & $1(5 \%)$ & $5(21.7 \%)$ & $6(9.4 \%)$ \\
\hline Asian & $0(0 \%)$ & $1(5 \%)$ & $1(4.3 \%)$ & $2(3.1 \%)$ \\
\hline Hispanic/Latino & $0(0 \%)$ & $0(0 \%)$ & $1(4.3 \%)$ & $1(1.6 \%)$ \\
\hline Mixed Race & $0(0 \%)$ & $0(0 \%)$ & $1(4.3 \%)$ & $1(1.6 \%)$ \\
\hline Other/Not Classified & $0(0 \%)$ & $0(0 \%)$ & $2(8.7 \%)$ & $2(3.1 \%)$ \\
\hline \multicolumn{5}{|l|}{ Current Relationship Status } \\
\hline Married or Committed Relationship & $6(28.5 \%)$ & $11(54.8 \%)$ & $15(65.2 \%)$ & $32(54.2 \%)$ \\
\hline Divorced or separated & $2(9.6 \%)$ & $0(0 \%)$ & $0(0 \%)$ & $2(3.3 \%)$ \\
\hline Single, not partnered & $13(61.9 \%)$ & $9(45 \%)$ & $8(34.8 \%)$ & $30(46.9 \%)$ \\
\hline \multicolumn{5}{|l|}{ Years of Education } \\
\hline Mean (SD) & $15.1(2.4)$ & $16.3(3.3)$ & $15.8(3.2)$ & $15.7(3.0)$ \\
\hline 12 or less & $3(14.3 \%)$ & $3(15 \%)$ & $2(8.7 \%)$ & $8(12.7 \%)$ \\
\hline $13-16$ & $13(61.9 \%)$ & $7(35 \%)$ & $12(52.2 \%)$ & $32(50 \%)$ \\
\hline $17-18$ & $5(23.8 \%)$ & $9(47.4 \%)$ & $9(39.1 \%)$ & $23(36.5 \%)$ \\
\hline Not Reported & $0(0 \%)$ & $1(5 \%)$ & $0(0 \%)$ & $1(1.6 \%)$ \\
\hline \multicolumn{5}{|l|}{ Verbal IQ } \\
\hline Mean (SD) & $121.0(11.6)$ & $125.6(12.0)$ & $120.6(13.9)$ & $122.2(12.6)$ \\
\hline
\end{tabular}

Note. Groups were not significantly different on age, years of education, or verbal IQ. \% (Percentage of each group as indicated). AN = current diagnosis of anorexia nervosa; $\mathrm{WR}=$ prior diagnosis of anorexia nervosa but currently weight-restored; $\mathrm{CN}=$ no history of anorexia nervosa. 


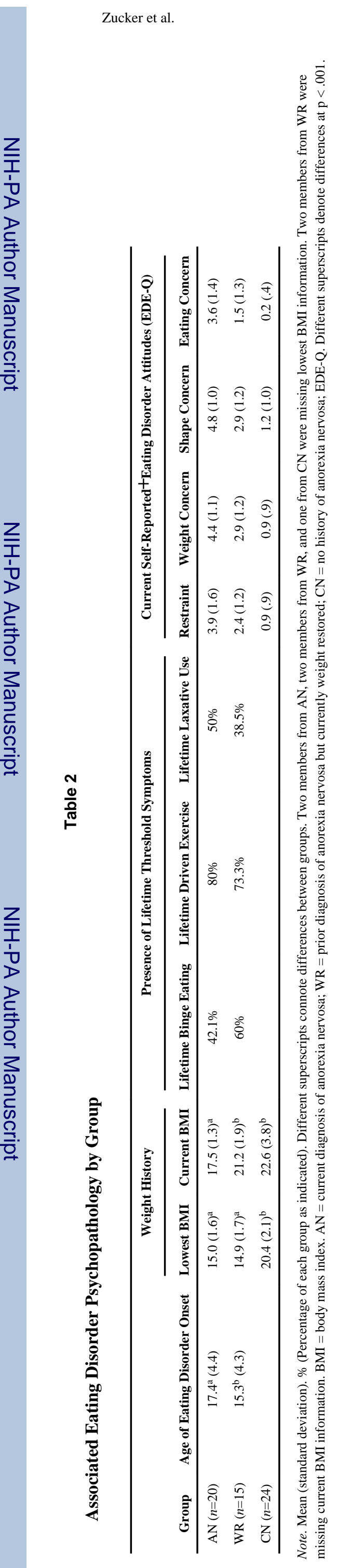

Ehav Res Ther. Author manuscriptat, avalable in PMC 2014 une 0 


\section{Table 3}

\section{Group Means on Sensory Profile}

\begin{tabular}{|c|c|c|c|c|}
\hline & \multicolumn{2}{|c|}{ Sensation } & \multicolumn{2}{|c|}{ Behavior } \\
\hline & Low Registration & Sensory Sensitivity & Sensation Avoidance & Sensation Seeking \\
\hline $\mathrm{AN}(\mathrm{n}=20)$ & $36.1(7.9) ; 20-48^{\mathrm{a}}$ & $44.1(7.7) ; 30-58^{\mathrm{a}}$ & $44.5(8.4) ; 21-55^{\mathrm{a}}$ & $51.3(7.9) ; 38-63$ \\
\hline WR $(n=15)$ & $34.1(5.8) ; 23-41^{\mathrm{ab}}$ & $43.7(8.0) ; 29-55^{\mathrm{a}}$ & $43.3(7.1) ; 30-55^{\mathrm{a}}$ & $52.1(4.2) ; 45-60$ \\
\hline $\mathrm{CN}(\mathrm{n}=24)$ & $29.3(6.5) ; 17-4^{\mathrm{bc}}$ & $34.3(8.8) ; 16-51^{\mathrm{b}}$ & $35.9(7.5) ; 19-49^{b}$ & 53.7 (7.3); 38-68 \\
\hline
\end{tabular}

Note: AN, Current Diagnosis of Anorexia Nervosa; WR, Prior Diagnosis of Anorexia Nervosa but weight-restored; CN - Control participants. Values represent Mean (STD); Range. Unique superscripts between groups denote pairwise differences between the groups at the family-wise error-rate at $\mathrm{p}<.008$. 